\title{
Quantifying the Sustainability Impact of Data Center Availability
}

\author{
Manish Marwah ${ }^{1}$, Paulo Maciel ${ }^{2}$, Amip Shah ${ }^{1}$, Ratnesh Sharma ${ }^{1}$, Tom Christian ${ }^{1}$, Virgilio Almeida ${ }^{4}$, Carlos Araújo ${ }^{2}$, \\ Erica Souza ${ }^{2}$, Gustavo Callou ${ }^{2}$, Bruno Silva $^{2}$, Sérgio Galdino ${ }^{2}$, Jose Pires ${ }^{3}$ \\ ${ }^{I}$ HP Labs, Palo Alto, CA, USA $\quad{ }^{2}$ UFPE, Recife, Brazil ${ }^{3}$ HP Brazil R\&D, Porto Alegre, Brazil ${ }^{4} U F M G$, Belo Horizonte, Brazil
}

\begin{abstract}
Data center availability is critical considering the explosive growth in Internet services and people's dependence on them. Furthermore, in recent years, sustainability has become important. However, data center designers have little information on the sustainability impact of data center availability architectures. In this paper, we present an approach to estimate the sustainability impact of such architectures. Availability is computed using Stochastic Petri Net (SPN) models while an exergy-based lifecycle assessment (LCA) approach is used for quantifying sustainability impact. The approach is demonstrated on real life data center power infrastructure architectures. Five different architectures are considered and initial results show that quantification of sustainability impact provides important information to a data center designer in evaluating availability architecture choices.
\end{abstract}

Categories and Subject Descriptors: C.4 [Performance of Systems]: Design studies, Modeling techniques, Reliability, availability, and serviceability.

General Terms: Measurement, Design, Economics, Reliability.

Keywords: Sustainability, availability, data center, power infrastructure, stochastic Petri net, life-cycle assessment.

\section{INTRODUCTION}

In recent years, there has been a significant growth in the number, size and power density of data centers. This has been fueled by paradigms such as software as a service (SaaS), cloud computing [1], and a whole gamut of Internet-based businesses, social networking sites and multimedia applications and services. With this growth, concern about the energy consumption and environmental sustainability of data centers has also grown. In fact, data centers in the U.S. consumed an estimated 61 billion kilowatt-hours $(\mathrm{kWh})$ of electricity $(1.5 \%$ of total U.S. consumption) in 2006 at the cost of $\$ 4.5$ billion, and expected to reach 100 billion $\mathrm{kWh}$ by 2011 [2]. These facts, in light of global warming, depletion of fossil fuels and rising energy costs, have brought sustainability of the IT infrastructure into sharp focus.

What do we mean by sustainability? In this context, environmentally sustainable data centers are those that are built using the least amount of the most appropriate materials and consume the least amount of the most appropriate sources of energy throughout their lifetime [25]. Life-cycle assessment (LCA) techniques [5][7], further discussed in Section 4, aim to

\footnotetext{
Permission to make digital or hard copies of all or part of this work for personal or classroom use is granted without fee provided that copies are not made or distributed for profit or commercial advantage and that copies bear this notice and the full citation on the first page. To copy otherwise, or republish, to post on servers or to redistribute to lists, requires prior specific permission and/or a fee.

GreenMetrics'09, June 15, 2009, Seattle, WA, USA

Copyright 2009 ACM 1-58113-000-0/00/0004 ..\$5.00.
}

implement this description of environmental sustainability.

Because of the increased dependence on Internet services, data center availability assumes greater significance. For companies that depend heavily on the Internet for their operations, service outages can be very expensive, easily running into millions of dollars per hour [3]. A widely used design principle in faulttolerance is to introduce redundancy to enhance availability. This allows use of inexpensive commodity components - having low availability individually - to provide high availability as an aggregate. This principle is widely practiced in the industry by companies that use commodity hardware and redundant architectures with software control to mask failures and increase availability. However, since redundancy leads to additional use of materials and energy, it is expected to have a negative impact on sustainability.

At present, data center designers have little information for quantitative evaluation of the sustainability impact of highavailability architectures. In fact, two different data center architectures with similar availability numbers may have very different sustainability impact. In order to make comparisons between different architectures and include sustainability considerations in decision making, it is necessary to quantify the sustainability impact of different design choices.

In this paper, we describe an approach to estimate both availability and sustainability of data center architectures. Availability is computed using stochastic Petri net (SPN) models [4] while a life-cycle assessment (LCA) [5][7] approach is used for quantifying sustainability impact. To demonstrate our approach, we apply it to data center power infrastructure architectures: we build availability models, estimate sustainability and evaluate various trade-offs for five different power infrastructure designs in a data center. For comparison, we also compute the total cost of ownership (TCO) of the architectures, which includes capital and operating expenses for their projected lifespan. Note that although we selected the power infrastructure for analysis in this paper, our approach applies equally well to any data center equipment.

In particular, this paper makes the following contributions:

- To the best of our knowledge, this is the first work to quantitatively consider the sustainability impact of date center availability architectures.

- We estimate availability of five different power infrastructure architectures of real data centers using stochastic Petri nets. The approach also enables estimating reliability, modeling active and passive redundancy mechanism and maintenance impact on dependability issues.

- Finally, we estimate the sustainability metrics corresponding to the architectures and compare them in terms of availability, sustainability and TCO. 


\section{DATA CENTER INFRASTRUCTURE}

A data center essentially consists of three sub-systems, in addition the building facility: (1) IT infrastructure, (2) power infrastructure, and (3) cooling infrastructure.

\subsection{IT Infrastructure}

The IT infrastructure consists of three main components - servers, networking equipment and storage devices. Server virtualization software may be used for hardware resource sharing, workload migration and consolidation. The storage devices (e.g. storage arrays) are typically connected through a storage area network (SAN). Servers may also connect to remote file systems on Network Attached Storage (NAS) over Ethernet. The network infrastructure is typically three-tier [15]: an access tier consisting of layer-2 switches into which individual servers plug in, an aggregation tier with layer 2-3 switches for connectivity within the data center; and a core tier with layer-3 routers that connect the data center to the Internet and the rest of the campus network. Software services are organized in a multi-tier architecture [14] with separate tiers for web servers, applications and database servers.

\subsection{Power Infrastructure}

The power infrastructure [14] [17] is responsible for providing uninterrupted, conditioned power at the correct voltage and frequency to the IT equipment hosted in data center racks. From the utility feed, typically, the power goes through step down transformers, transfer switches, uninterruptible power supplies (UPS), PDUs, and finally to rack power strips. For fault-tolerance, many elements on the path of power are duplicated depending on the data center tier [13]. The UPS condition power and provide backup power in case of short outages. Generators or other local power sources may be used for longer outages, or even for satisfying a part of the total power demand on a regular basis.

\subsection{Cooling Infrastructure}

Virtually all of the power that is fed into the IT infrastructure is transformed into heat. The data center cooling infrastructure is responsible for extracting that heat to prevent the IT equipment from overheating. Typically, multiple CRAC units transfer heat dissipated by IT equipment to a chilled water distribution system. Chillers extract heat from the chilled water system and reject it to the environment through cooling towers or heat exchangers. The cooling infrastructure may account for $10-50 \%$ of the total power consumption of the data center [26].

\section{AVAILABILITY MODELING}

This section describes the modeling approach adopted for quantifying system availability. The proposed modeling approach using Stochastic Petri Nets (SPN) is generic enough to model a wide variety of complex redundancy mechanisms and scenarios, and, is applicable to any equipment in a data center. The introduction of timing concepts into Petri Nets (PN) models were proposed by Ramchandani [18]. Molloy [20] and Florin [19] proposed PN models in which stochastic timing was considered. These works opened the possibility of connecting PN theory and stochastic modeling. Today, these models and their extensions are generically named Stochastic Petri Nets (SPN) [22].

SPNs allow incorporation of dependencies between devices, subsystems, complex active-redundant mechanisms and resource constraints, such as, maintenance team size and skills. System dependencies are represented through arcs between places (local states) and transitions, and marking dependent parameters.
Transitions are only enabled if their input place markings enable a transition firing. The use of local states allows the representation of complex dependency interactions between sub-systems. Transition delays and weights might also be dependent on place markings.

The evaluation methods for solving SPN involve Markov chain generation or discrete event simulation depending on system complexity and size, computer memory available, metrics to be computed and time available for providing the results.

The SPN modeling is based on a parameterized basic model library that allows us to represent a large number of passive and active redundant mechanisms as well as expolynomial distributions [21]. The simplest model of the library is called a simple component. Such a component is shown in Figure 1 and is characterized by the absence of redundancy. This component has two parameters (not shown in the figure), namely X_MTTF and X_MTTR, which represent delays associated to transitions $\mathrm{X}$ Failure and $\mathrm{X}$ Repair, respectively. Both transitions are exponentially distributed, have delays that are not marking dependent, and have single-service concurrency policies. If place $\mathrm{X}$ Rel Flag is marked (one token), the arc weight from place $\mathrm{X}_{-}$OFF $\bar{F}$ to transition $\mathrm{X}$ _Repair is equal to 2, otherwise the arc weight is $1-$ this arc weight is marking dependent. Hence, the metric expression $\mathrm{P}\{\# \mathrm{X} \mathrm{ON}=1\}^{1}$ provides the component's availability when \#X_Rel_Flag $=0$, and the component's reliability when \#X_Rel_Flag $=1$.

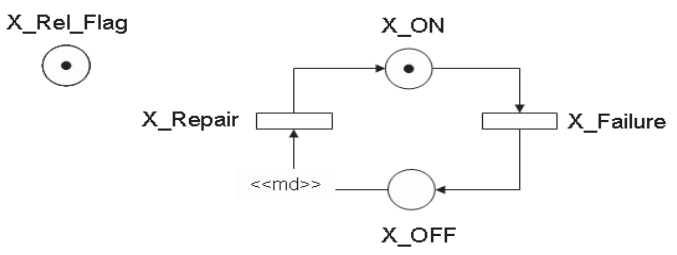

Figure 1: Simple component model.

\section{SUSTAINABILITY METRICS}

Life-cycle assessment (LCA) is a common approach to quantify environmental sustainability [5][7]. Here we use a LCA-based approach to estimate the sustainability impact of equipment in terms of its lifetime exergy (available energy) consumption. It should be noted that while the proposed approach is applied here for analysis of the data center power infrastructure (Section 5), the model is sufficiently general to be applied to any data center equipment including IT and cooling infrastructures.

\subsection{Life-cycle assessment}

In such an approach, an aggregated inventory of the different materials and processes involved across the life-cycle of the system - from extraction of raw materials and manufacturing through operation and end-of-life - is compiled. This inventory is then translated into different environmental impacts through a set of standardized or predetermined impact factors. For example, by knowing the total electricity use in $\mathrm{kWh}$ across the life-cycle (inventory) and the average composition of the electricity mix in terms of $\mathrm{kg} \mathrm{CO}_{2}$-equivalent per $\mathrm{kWh}$ (impact factor), an estimate of the global warming potential (environmental impact) in $\mathrm{kg}$ $\mathrm{CO}_{2}$-equivalent can be calculated. Additional details regarding LCA can be found in the literature [5][7].

${ }^{1} \mathrm{P}\left\{\# \mathrm{X} \_\mathrm{ON}=1\right\}$ indicates probability that place $\mathrm{X} \_\mathrm{ON}$ stores one token. 
Collecting the necessary inventory data for compiling a detailed process-based LCA can be time-consuming and costly owing to the vast amount of information required and the difficulty of obtaining such information across complex and diverse supply chains. Expeditious LCA approaches - such as streamlined LCA [8] and economic input-output (EIO) LCA [9] - have been developed as alternatives. In the present work, we utilize a hybrid EIO-LCA and process-based approach to quantify the net environmental impact in terms of lifetime exergy consumption (described below). The approach, which is similar to that utilized by Shah et al. [10][11], essentially divides the life-cycle into two phases: (1) embedded phase, which involves all impacts related to product design decisions (including material extraction, manufacturing and supply chain impacts, as well as end-of-life); and, (2) operational phase, which involves all impacts related to decisions during product use (such as operational and maintenance cycles). For the embedded phase, an EIO-LCA model is developed that quantifies the environmental impacts in terms of average sectoral compositions as a function of crosssectoral economic activity. For the operational phase, a detailed operational model is constructed that takes into account the individual device efficiencies, uptime, and runtime power consumption. Additional details are available in the work of Shah et al. [10][11].

\subsection{Exergy}

In the present work, we choose to quantify the environmental impact in terms of the thermodynamic metric of exergy (also called usable available energy), which represents the part of a system that can be converted into useful work and has the same units as energy (Joules). This metric was chosen because it presents a unified view of the total material and energy impact, as well as the potential to recover any useful work from exhaust streams (such as the heat dissipated from the device). As discussed by Lettieri et al. [6], for most IT systems and related infrastructural components, such a life-cycle exergy analysis (LCEA) provides a reasonable compromise between the amount of time required in the analysis and the relative accuracy of the analysis. The underlying foundation behind this thesis lies in the recognition that IT systems, like many other classes of workconsuming devices, tend to be 'exergy intensive' - namely, their environmental footprint consists mostly of impacts related to the measurable consumption of materials and energy. Because the metric of exergy loss by definition quantifies the magnitude of irreversibilities related to transitions of materials and energy from one form to another, for systems where material- or energy-related impacts tend to dominate, an LCEA often indirectly reflects broader environmental impacts such as toxicity, ecosystem degradation etc. A more thorough discussion of the applicability of exergy loss for such broad-ranging sustainability assessments can be found in the work of Shah and Meckler [23].

It should be clarified that we do not suggest an exergy-based approach as being sufficient for quantifying environmental impacts in all cases. Rather, we suggest that such an approach is appropriate to obtain a high-level first-order approximation of the environmental impacts. In a more general sense, we propose that for exergy-intensive systems, including many IT and workconsuming systems - an exergy-based approach be implemented as the first step of a broader sustainability assessment. Depending on the results from LCEA, more detailed models can be constructed for any life-cycle phases or sub-systems as desired. Shah et al. [24] have shown the viability of such a tiered LCEA approach for assessing the environmental impacts of IT systems, including those typically categorized in a traditional LCA (such as human health, ecosystem quality, and resource consumption).

\section{CASE STUDY: DATA CENTER POWER INFRASTRUCTURE}

As a proof of concept, we demonstrate our approach on real data center power delivery infrastructure. Note that the techniques described above are generic enough to be applied to other data center infrastructure elements as well as more complex redundancy mechanisms. We consider the power infrastructure starting at the UPS up to the IT rack, consisting of: UPS, a stepdown transformer, an electrical subpanel, and, a rack power strip. To enhance availability, one or more of these components may be duplicated. We consider five architectures with increasing redundancy, such that each successive architecture has an additional component duplicated. For each architecture, we estimate: (1) availability using a SPN model; and, (2) lifetime exergy as described in Section 4.2.

\subsection{Availability Models}

Figure 2 shows the baseline architecture (A1), with no redundant components. The failure and recovery rates for the components were taken from [17] and [12]. The proposed SPN model that represents this architecture is shown in Figure 3. The MTBF $i(i=$ 1..4) transitions represent the mean time between failure related to UPS, step-down transformer (SDT), Subpanel, and rack power strip respectively, while MTTR $i(i=1 . .4)$ transitions represent the mean time to recover for each component respectively. Furthermore, the UPS ON, SDT ON, SubPanel ON and Powerstrip_ON places represent the operational states of components and the corresponding OFF states represent the failure states. Table 1 shows the transition guard expressions related to the model of architecture A1.

In the second architecture (A2), the UPS is duplicated in a hot standby arrangement. Note that this architecture requires use of a static transfer switch (STS) as shown in Figure 4 so that either UPS can connect to the transformer. The SPN model of A2 is similar to that of A1 but with an additional component for the backup UPS. Similarly, we consider architectures A3, A4 and A5 where the transformer, subpanel and the rack power strip are also duplicated, respectively. Note that in architecture A5, where all four components are replicated including the power strip, an STS is not required since modern data centers have dual corded systems.

The steady state availability results from the SPN models of each of the five architectures are plotted in Figure 5 and also summarized in Table 2. If $\mathrm{A}$ is the availability, the number of nines of availability is calculated as follows:

Number of nines $=-\log (1-\mathrm{A})$

Table 1: Guard expressions for SPN model of architecture A1

\begin{tabular}{|c|c|}
\hline Transition & Guard Expressions \\
\hline Failure & $\begin{array}{l}((\# \text { UPS_ON=0)OR }(\# \text { SDT_ON=0)OR(\#SubP } \\
\text { anel_ON=0)OR(\#Powerstrip_ON=0) })\end{array}$ \\
\hline Repair & $\begin{array}{l}((\# \mathrm{UPS} \text { ONN }>0) \text { AND }(\# \mathrm{SDT} \text { ON }>0) \mathrm{AND}(\# \mathrm{~S} \\
\text { ubPanel_ON }>0) \text { AND }(\# \text { Powerstrip_ON }>0))\end{array}$ \\
\hline
\end{tabular}




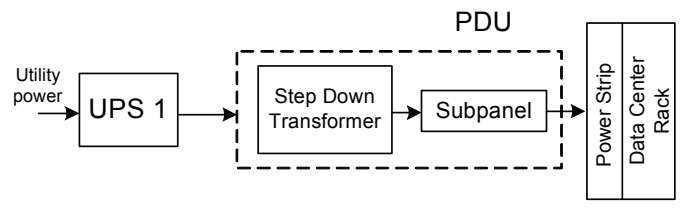

Figure 2: Baseline power delivery architecture (A1)

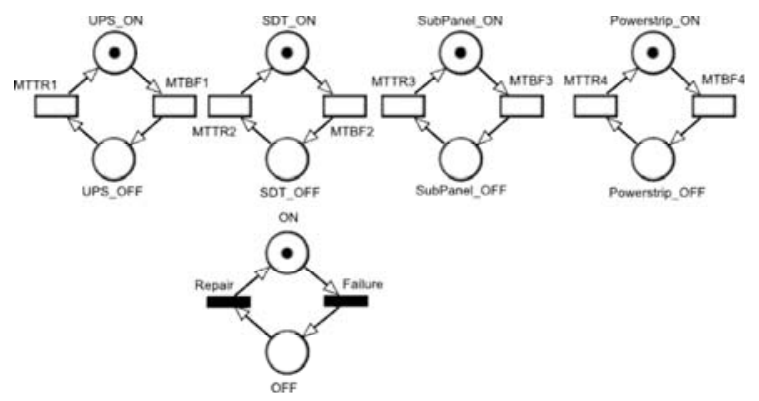

Figure 3: SPN model for architecture A1

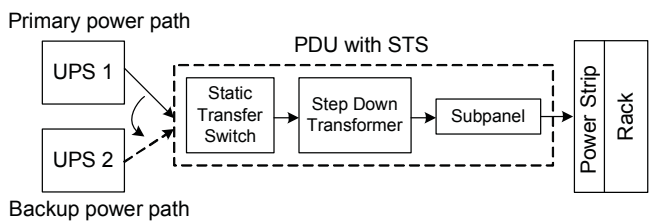

Figure 4: Architecture A2 with redundant UPS and STS.

Table 2: Summary of results corresponding to availability, sustainability metric and total cost for the five architectures.

\begin{tabular}{|l|l|l|l|}
\hline \multicolumn{1}{|c|}{ Architecture } & $\begin{array}{l}\text { Availability (\%) } \\
\text { (number of 9's) }\end{array}$ & $\begin{array}{l}\text { Lifetime } \\
\text { exergy } \\
\text { (GJ) }\end{array}$ & $\begin{array}{l}\text { TCO } \\
\text { (USD) }\end{array}$ \\
\hline \hline A1 (U-T-P-S) & $99.98494(3.8)$ & 32.9 & 5,517 \\
\hline A2 (2U-STS-T-P-S) & $99.985944(3.9)$ & 38.6 & 9,985 \\
\hline A3 (2U-2T-STS-P-S) & $99.997126(4.5)$ & 39.0 & 10,535 \\
\hline A4 (2U-2T-2P-STS -S) & $99.999905(6.0)$ & 39.4 & 10,735 \\
\hline A5 (2U-2T-2P-2S) & $99.999997(7.6)$ & 36.9 & 10,067 \\
\hline
\end{tabular}

Key: U - UPS; T - Transformer; P - Subpanel; S - Rack power strip; STS - Static transfer switch

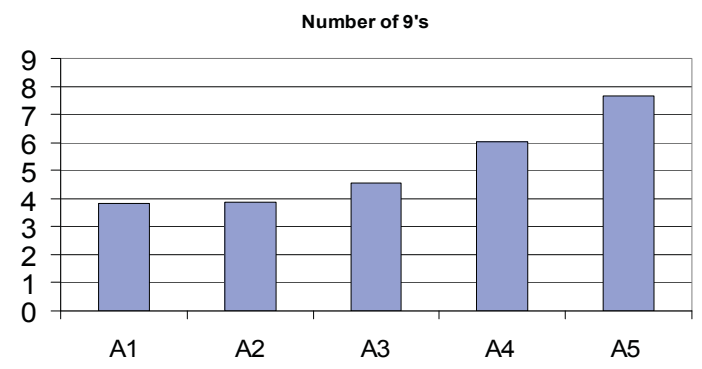

Figure 5: Availability results from the SPN models of the five architectures.

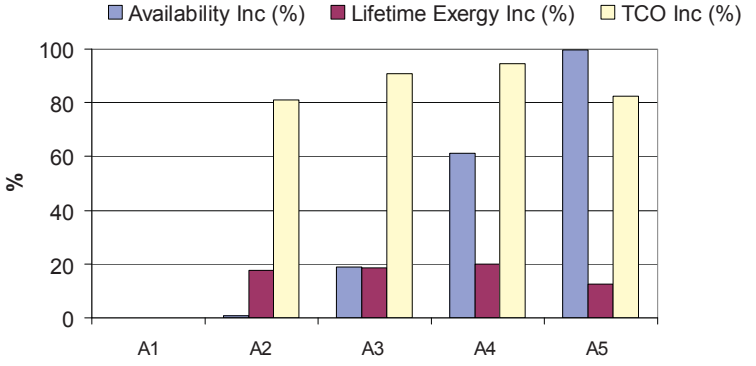

Figure 6: Percentage change over baseline (A1) in availability, lifetime exergy consumption and total cost.

As expected, availability increases with increasing redundancy. However, note that the availability increase from A1 to A2 is minimal since although the UPS is duplicated, a STS, which is a single point of failure, is also introduced. The number of nines doubles from A1 to A5, as expected, since A5 is essentially two instances of A1 connected in parallel. The availability of these architectures was also computed using reliability block diagrams (RBD) and the results obtained were almost identical.

\subsection{Sustainability and TCO Models}

The hybrid LCA approach, described in Section 4, was used to compute the sustainability metric (lifetime exergy consumption) for all the architectures. It quantifies, in joules, the total available energy consumed by the power delivery infrastructure across its lifespan (including during extraction of raw materials, processing and manufacturing, to operation and end-of-life). It can also be converted into a $\mathrm{kg}-\mathrm{CO}_{2}$ equivalent to determine its carbon footprint. The results are summarized in Table 2. The lifetime exergy shows a generally increasing trend with increasing redundancy in the power delivery infrastructure. There is a marked increase $(\sim 18 \%)$ from A1 to A2 since a UPS and STS are added. From A2 to A4, it is almost steady with only a $2 \%$ increase as the additional hardware has a small sustainability footprint. However, from A4 to A5, it in fact decreases since although an additional rack power strip is added in A5, the STS used in A4 is no longer needed.

Also shown in Table 2 are estimates of the approximate total cost of ownership (TCO) for the five architectures. These are based on the retail price of the components and cost of operations as a fraction of total data center costs over a period of five years. These are computed as a point of comparison to see if a cost effective design is also more sustainable.

\subsection{Comparison of Architectures}

Figure 6 shows the percentage change (over A1) in availability, lifetime exergy and TCO for architectures A2 through A5. A big jump in cost from A1 to A2 reflects the high cost of UPS as compared to the other components. The changes in lifetime exergy are primarily due to additional hardware, since the difference in operational power consumption between architectures is negligible.

Sustainability and availability. How does sustainability change with availability? Intuitively, it is believed that as availability is increased by adding more redundancy, the sustainability decreases due to use of more hardware. However, from Figure 6, it can be seen that as availability increases significantly from A2 to A3 (about 15\%) and then A3 to A4 (about 33\%), the sustainability impact increase is negligible. Thus, minimizing redundancy may 
not always lead to optimal sustainability. The sustainability impact decreases from A4 to A5; this is because the STS is not required in A5. Note that redundancy increases from A4 to A5.

Sustainability and cost. How does sustainability change with cost? From Figure 6, it can be seen that although both total cost and sustainability increase slightly from A2 to A4, they do not always change together. While A2 has a slightly lower cost, A5 has lower exergy consumption. Thus, optimizing based on cost will not necessarily lead to optimal sustainability.

Figure 7 shows the lifetime exergy cost per nine of availability for the five architectures. Thus with the exception of Architecture A2, the sustainability impact per unit availability keeps decreasing from A1 to A5.

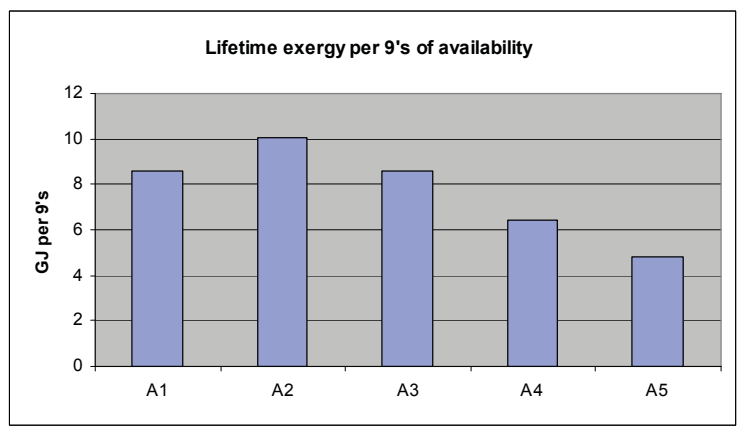

Figure 7 Sustainability impact for each architecture per number of nines of availability

\section{CONCLUSIONS AND FUTURE WORK}

The importance of environmental sustainability necessitates its consideration in design of highly available data centers. However, existing metrics used by data center designers are not a true indicator of sustainability. Total cost or the amount of redundancy used in a data center may not always correlate to sustainability.

This paper consists of two main pieces of work: (1) application of stochastic Petri net models to estimate availability under a wide variety of conditions; Petri nets allow device and sub-system dependencies, impact of maintenance teams and resource constraints to be considered; (2) application of a hybrid-LCA model to estimate lifetime exergy, a good indicator of first order sustainability impact of data center equipment [6]. We demonstrated our approach on five power infrastructure architectures for which availability and sustainability metric were computed. The results show that a sustainability metric can provide useful information to a data center designer in making architecture choices.

A limited part of a data center and a small number of architectures were considered here. In order to effectively explore the entire design space in a data center that satisfies given availability requirements, an automated mechanism would be necessary. Quantification of the sustainability impact would allow it to be included in user-defined optimization criteria. Furthermore, more detailed LCA models can be considered for broader sustainability assessment.

\section{ACKNOWLEDGMENTS}

The authors wish to thank Cullen Bash, Chandrakant Patel and Martin Arlitt for their feedback.

\section{REFERENCES}

[1] M. Armbrust, et al., "Above the Clouds: A Berkeley View of Cloud Computing”, U.C. Berkeley White Paper, Feb. 2009.

[2] U.S. Environmental Protection Agency, "Report to congress on server and datacenter energy efficiency," Public Law 109-431, 2007.

[3] D. A. Patterson, "A Simple Way to Estimate the Cost of Downtime," LISA 2002, pp 185-188.

[4] G. Balbo, "Introduction to Stochastic Petri Net," Lectures on Formal Methods and Performance Analysis. First EEF/Euro Summer School on Trends in Computer Science, Bergen Dal, The Netherlands, July 3-7, 2000: Revised Lectures, 2001.

[5] H. Baumann, A.-M. Tillman, The Hitch Hiker's Guide to LCA, Studentlitteratur AB, Sweden, 2004.

[6] D.J. Lettieri, et al., "Lifetime Exergy Consumption as a Sustainability Metric for Information Technologies, ” IEEE Intl. Symp. Sus. Sys. Tech. (ISSST), Phoenix, AZ, 2009.

[7] International Organization for Standardization (ISO), "ISO 14064: Environmental Management: Life Cycle Assessment - Principles and Framework," Technical Document, 2006.

[8] T.E. Graedel, Streamlined Life-Cycle Assessment, Prentice Hall: New Jersey, 1998.

[9] C.T. Hendrickson, et al., Environmental Life Cycle Assessment of Goods and Services: An Input-Output Approach, Resources for the Future: Washington DC, 2006.

[10] A. Shah, et al., "The Environmental Impact of Data Centers," ASME InterPACK, San Francisco, CA, 2009.

[11] A. Shah, C. Patel, "Designing Environmentally Sustainable Cooling Solutions using Exergo-Thermo-Volumes," Int. J. Energy Res., 2009.

[12] V. Avelar, "Comparing Availability of Various Rack Power Redundancy Configurations," APC White Paper \#48, 2003.

[13] W. P. Turner IV, J. H. Seader, and K. G. Brill. "Tier classifications define site infrastructure performance, » White Paper, The Uptime Institute, 2006.

[14] X. Fan, W. Weber, and L.A. Barroso, "Power provisioning for a warehousesized computer," Proc. 34th Annual Int. Symp. Comp. Arch. (ISCA), San Diego, CA, June 2007.

[15] L. A. Barroso, J. Dean, and U. Holzle, "Web search for a planet: The google cluster architecture," IEEE Micro, vol. 23, no. 2, pp. 22-28, 2003.

[16] M. Al-Fares, A. Loukissas, A. Vahdat, "A Scalable, Commodity Data Center Network Architecture,” SIGCOMM 2008.

[17] IEEE Gold Book 473, IEEE Gold Book 473, Design of Reliable Industrial and Commercial Power Systems

[18] C. Ramchandani, "Analysis of Asynchronous Concurrent Systems by Timed Petri Nets," PhD thesis, MIT, Cambridge, MA, 1974

[19] G. Florin, S. Natkin. "Matrix Product Form Solution for Closed Synchronized Queueing Networks.” Proc. 3rd Intl. Workshop on Petri Nets and Performance Models, pp. 29-39, 1989

[20] M. K. Molloy, "On the Integration of Delay and Throughput Measures in Distributed Processing Models," PhD thesis, UCLA, Los Angeles, CA, 1981

[21] K. Trivedi, Probability and Statistics with Reliability, Queuing, and Computer Science Applications, John Wiley and Sons, 2006.

[22] M. Ajmone Marsan, G. Balbo, G. Conte, S. Donatelli and G. Franceschinis Modelling with Generalized Stochastic Petri Nets. Wiley Series in Parallel Computing John Wiley and Sons, 1995.

[23] A. Shah, M. Meckler, "An Exergy-Based Framework for Assessing Sustainability of IT Systems,” Proc. ASME Energy Sustainability Conference (ES2009), San Francisco, CA [in press], 2009.

[24] A. Shah, C. Patel, V. Carey, "Exergy-Based Metrics for Sustainable Design," Proc. 4th Intl. Exergy, Energy, and Environment Symp. (IEEES-4), Sharjah, UAE, 2009.

[25] C.E. Bash, C.D. Patel, A.J. Shah, R.K. Sharma, "The Sustainable Information Technology Ecosystem.” In Proceedings of the 11 th Intersociety Conference on Thermal and Thermomechanical Phenomena in Electronic Systems (ITherm '08), May 2008.

[26] C.L. Belady. In the data center, power and cooling costs more than the IT equipment it supports. Electronics Cooling, 13(1), Feb 2007. 